\title{
Electron scattering mechanisms in GZO films grown on a-sapphire substrates by plasma-enhanced molecular beam epitaxy
}

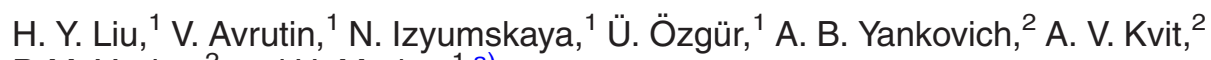 \\ P. M. Voyles, ${ }^{2}$ and H. Morkoç ${ }^{1, a)}$ \\ ${ }^{1}$ Department of Electrical and Computer Engineering, Virginia Commonwealth University, Richmond, \\ Virginia 23284, USA \\ ${ }^{2}$ Department of Materials Science \& Engineering, University Wisconsin-Madison, Madison, Wisconsin 53706 USA
}

(Received 22 August 2011; accepted 23 April 2012; published online 24 May 2012)

\begin{abstract}
We report on the mechanisms governing electron transport using a comprehensive set of $\mathrm{ZnO}$ layers heavily doped with Ga (GZO) grown by plasma-enhanced molecular-beam epitaxy on a-plane sapphire substrates with varying oxygen-to-metal ratios and Ga fluxes. The analyses were conducted by temperature dependent Hall measurements which were supported by microstructural investigations as well. Highly degenerate GZO layers with $n>5 \times 10^{20} \mathrm{~cm}^{-3}$ grown under metalrich conditions (reactive oxygen-to-metal ratio $<1)$ show relatively larger grains $(\sim 20-25 \mathrm{~nm}$ by $\mathrm{x}$-ray diffraction) with low-angle boundaries parallel to the polar $c$-direction. For highly conductive GZO layers, ionized-impurity scattering with almost no compensation is the dominant mechanism limiting the mobility in the temperature range from 15 to $330 \mathrm{~K}$ and the grain-boundary scattering governed by quantum-mechanical tunnelling is negligible. However, due to the polar nature of $\mathrm{ZnO}$ having high crystalline quality, polar optical phonon scattering cannot be neglected for temperatures above $150 \mathrm{~K}$, because it further reduces mobility although its effect is still substantially weaker than the ionized impurity scattering even at room temperature (RT). Analysis of transport measurements and sample microstructures by $\mathrm{x}$-ray diffraction and transmission electron microscopy led to a correlation between the grain sizes in these layers and mobility even for samples with a carrier concentration in the upper $10^{20} \mathrm{~cm}^{-3}$ range. In contrast, electron transport in GZO layers grown under oxygen-rich conditions (reactive oxygen-to-metal ratio $>1$ ), which have inclined grain boundaries and relatively smaller grain sizes of $10-20 \mathrm{~nm}$ by $\mathrm{x}$-ray diffraction, is mainly limited by compensation caused by acceptor-type point-defect complexes, presumably $\left(\mathrm{Ga}_{\mathrm{Zn}}-\mathrm{V}_{\mathrm{Zn}}\right)$, and scattering on grain boundaries. The GZO layers with $\mathrm{n}<10^{20} \mathrm{~cm}^{-3}$ grown under metal-rich conditions with reduced Ga fluxes show a clear signature of grain-boundary scattering governed by the thermionic effect in the temperature-dependent mobility but with much higher RT mobility values compared to the samples grown under oxygen-rich conditions [ $\left.34 \mathrm{vs} .7 .5 \mathrm{~cm}^{2} / \mathrm{V} \cdot \mathrm{s}\right]$. Properties of GZO layers grown under different conditions clearly indicate that to achieve highly conductive GZO, metal-rich conditions instead of oxygen-rich conditions have to be used. (C) 2012 American Institute of Physics. [http://dx.doi.org/10.1063/1.4720456]
\end{abstract}

\section{INTRODUCTION}

$\mathrm{ZnO}$ doped with $\mathrm{Ga}$ or $\mathrm{Al}(\mathrm{GZO}$ or $\mathrm{AZO})$ has recently gained a great deal of interest as a transparent conducting oxide (TCO) layer in conjunction with light emitters and photovoltaic devices due to its low cost, abundant material resources, and non-toxicity. ${ }^{1}$ TCO films with high conductivity resulting from the high mobility rather than from high carrier concentration are desired in photovoltaics because of the unwanted free carrier absorption in the IR spectral range while retaining conductivity owing to relatively high mobility. ${ }^{1}$ The reported mobility for GZO and AZO films grown by different techniques scatters from as low as $\sim 5$ to as high as $70 \mathrm{~cm}^{2} / \mathrm{V} \cdot \mathrm{s}$ for electron carrier concentrations exceeding $10^{20} \mathrm{~cm}^{-3}$. It is generally accepted that the wide dispersion in the mobility is due to the differences in materials quality since the substrate temperature, film thickness, annealing conditions, and reactant compositions all affect

\footnotetext{
${ }^{a)}$ Email: hmorkoc@vcu.edu. Telephone: +1 804827 3765. Fax: +1 804828 4269.
}

the electrical properties. ${ }^{2}$ Furthermore, explanations of electron transport in heavily doped $\mathrm{ZnO}$ are still controversial. From a theoretical point of view, the grain-barrier model employed by Seto $^{3}$ and Bruneaux et al. $^{4}$ suggests that the grain boundary scattering is negligible for heavily doped TCOs since the barriers at the grain boundaries are thin enough for electrons to tunnel. The contribution of the grain boundaries to electron scattering is believed to be entirely screened out by the contribution of the bulk of the crystallites. ${ }^{4}$ The aforementioned is supported by Ellmer and Mientus, ${ }^{5}$ Minami et al. ${ }^{6}$ Steinhauser et al., ${ }^{7}$ and Ruske et al. ${ }^{8}$ Minami et al. ${ }^{6}$ have concluded that grain boundary scattering is mainly dominant in AZO films with carrier concentrations in the range $10^{19}-10^{20} \mathrm{~cm}^{-3}$ while the ionized impurity scattering is dominant in layers with carrier concentrations in the range $10^{20}-10^{21} \mathrm{~cm}^{-3}$. Steinhauser et al..$^{7}$ concluded that the grain boundaries do not limit the conductivity for carrier concentrations exceeding $1 \times 10^{20} \mathrm{~cm}^{-3}$ based on the comparison between Hall mobility and optical mobility for boron-doped $\mathrm{ZnO}$. By comparing the Hall mobility and the optical mobility, Ruske et al. ${ }^{8}$ have argued that grain 
boundaries do not limit the conductivity only for $\mathrm{ZnO}$ :Al films when the electron concentration is above $\sim 5 \times 10^{20} \mathrm{~cm}^{-3}$. On the other hand, Robbins et al. ${ }^{9}$ concluded that the grain boundary scattering and ionized impurity scattering contribute nearly equally to the overall mobility based on the analysis of nanocrystalline GZO with a carrier concentration of $5.5 \times 10^{20}$ $\mathrm{cm}^{-3}$ and an average grain size of $80 \mathrm{~nm}$. Ahn et al. ${ }^{10}$ suggested that the grain boundary scattering limits the mobility in GZO with carrier concentrations above $10^{20} \mathrm{~cm}^{-3}$ in the temperature range 190-300 K.

It must be pointed out here that, for $\mathrm{ZnO}$-based TCOs with dominating ionized impurity scattering, the negative effect of donor compensation with acceptor-type defects on the electron mobility is very strong and can be clearly seen experimentally, as was demonstrated by Look et al. ${ }^{11}$ The compensation mechanisms in GZO have been found ${ }^{12}$ to be strongly dependent on growth conditions of the material. Using a hybrid functional theory together with experimental measurements, Demchenko et al. ${ }^{12}$ have demonstrated that, for GZO growth under metal-rich growth conditions, the $\mathrm{Ga}_{\mathrm{zn}}$ donors have the lowest formation energy within a wide range of electron concentrations including the highly degenerate material. Thus, a low compensation level is expected. On other hand, the formation energy of $\left(\mathrm{Ga}_{\mathrm{Zn}}-\mathrm{V}_{\mathrm{Zn}}\right)$ acceptor complexes decreases with increasing electron concentration in GZO grown under oxygen-rich growth conditions and becomes lower than that of the $\mathrm{Ga}_{\mathrm{zn}}$ donors in the degenerate material (at $n>5 \times 10^{18} \mathrm{~cm}^{-3}$ ). Consequently, GZO films grown under oxygen-rich conditions have lower mobility partially due to higher compensation induced by $\left(\mathrm{Ga}_{\mathrm{Zn}}-\mathrm{V}_{\mathrm{Zn}}\right)$ acceptor complexes.

The main, and arguably the remaining, issue is the nature of mechanisms that limit the mobility in heavily doped $\mathrm{ZnO}$. Molecular-beam epitaxy (MBE) technique allows precise control over the process parameters, such as the substrate temperature and fluxes of the constituents and dopants, and therefore, can tailor the material parameters in an effort to find any relationship between its structural and transport characteristics. In order to address the above mentioned scattering mechanisms, we undertook investigations of electron transport in GZO layers with carrier concentrations varying from $\sim 6 \times 10^{18}$ to $9 \times 10^{20} \mathrm{~cm}^{-3}$, which were grown by RF plasma-enhanced $\mathrm{MBE}$ on a-plane sapphire substrates under a wide range of conditions.

\section{BRIEF DISCUSSION OF SCATTERING MECHANISMS}

Let us first discuss briefly the major scattering mechanisms which govern the electron transport in $\mathrm{ZnO}$-based TCO. These mechanisms include carrier scattering by polar optical phonons (POP), ionized impurities, and extended defects (mainly grain boundaries). It should be pointed out that phonon scattering through deformation potential (acoustic phonon scattering) is negligible compared with POP scattering in $\mathrm{ZnO}$ due to its polar nature ${ }^{13,14}$ which also applies to other polar materials. For undoped $\mathrm{ZnO}$, the room-temperature (RT) mobility limited by phonon scattering through piezoelectric potential (piezoelectric scattering) was calculated by Jung et al. ${ }^{15}$ to be much higher than $10000 \mathrm{~cm}^{2} / \mathrm{V} \cdot \mathrm{s}$, where $\varepsilon_{0}=$
8.12 and $\varepsilon_{\infty}=3.72$, piezoelectric coefficient $=0.21$, effective mass $=0.318 \mathrm{~m}_{0}$. Here the formula and constants are consistent with other reports in the literature. To calculate the mobility limited by piezoelectric scattering for heavily doped $\mathrm{ZnO}$, only the effective mass need to be replaced with an increased value corresponding to the doping level. However, this correction does not change relative mobilities limited by the aforementioned mechanisms and POP scattering still has substantially larger effect on electron mobility. Therefore, POP scattering is the only phonon-based scattering mechanism to be considered. Others such as dislocation scattering and neutral impurity scattering which are rarely used in transparent conductors ${ }^{13,16}$ can be neglected for heavily doped GZOs too because the mobility limited by each of them is at least $\sim 5000 \mathrm{~cm}^{2} / \mathrm{V} \cdot \mathrm{s}$ in the temperature range $15-330 \mathrm{~K}$. The temperature dependence for the above mentioned scatterings can be found elsewhere. ${ }^{15}$

\section{POP scattering}

POP scattering is important in polar materials at temperature near or above RT. As calculated by Jung et al. ${ }^{15}$ for high quality undoped $\mathrm{ZnO}$ grown by MBE, the effect of POP scattering is much stronger than other scattering mechanisms, including deformation potential scattering, piezoelectric scattering, and dislocation scattering. The temperature dependent mobility limited by POP scattering is given by ${ }^{15,17}$

$$
\begin{aligned}
\mu_{\text {pop }}\left[\mathrm{cm}^{2} / V \cdot s\right]= & 0.199 \times(T / 300)^{1 / 2} \\
& \times\left(\frac{e}{e^{*}}\right)^{2} \times\left(m_{0} / m *\right)^{3 / 2} \times\left(10^{22} M[g]\right) \\
& \times\left(10^{23} \nu_{a}\left[\mathrm{~cm}^{3}\right]\right) \times\left(10^{-13} \omega\left[s^{-1}\right]\right) \\
& \times\left(e^{\hbar \omega / k_{B} T}-1\right) \times G\left(\frac{\hbar \omega}{k_{B} T}\right)
\end{aligned}
$$

where $e^{*}$ is the Callen effective charge, $M$ is the reduced mass, $V_{a}$ is the volume of the unit cell, and $m^{*}$ is the effective mass of electron. The Ehrenreich's function $\mathrm{G}\left(\hbar \omega / k_{B} T\right)$ accounting for the screening effect ${ }^{17}$ is a slowly varying function of temperature $T$, with $\hbar \omega$ being the LO phonon energy, which is $72 \mathrm{meV}$ in the case of $\mathrm{ZnO}$. Note that $k_{B}$ is Boltzmann constant. For degenerate materials, when the Fermi level is located deeply in the conduction band, the POP scattering may become even more important because of increase in the electron effective mass caused by nonparabolicity of the conduction band.

\section{Ionized impurity scattering}

Ionized impurity scattering is a very important scattering mechanism in doped materials. According to the BrooksHerring formula for degenerate materials simplified by Look et al., ${ }^{11}$ the ionized impurity limited mobility in partially compensated films can be expressed as $\mu_{i i}=\mu_{\max }(1-K) /$ $(1+K)$ where $K$ is the compensation ratio and $\mu_{\text {max }}$ represents the maximum attainable mobility limited by ionized impurity scattering (mobility at $0 \mathrm{~K}$ ) in samples with no compensation. $\mu_{\max }$ as a function of electron concentration $n$ 
is given in the following equation with $Z$ being the ionization charge in units of $e$ and $\varepsilon_{0}$ being the dielectric constant

$$
\begin{array}{r}
\mu_{\max }=\frac{24 \pi^{3} \varepsilon_{0}^{2} \hbar^{3}}{Z^{2} e^{3} m^{* 2}} \frac{1}{\ln [1+y(n)]-\frac{\dot{y}(n)}{1+y(n)}} \\
\text { with } \quad y(n)=\frac{3^{1 / 3} 4 \pi^{8 / 3} \varepsilon_{0} \hbar^{2} n^{1 / 3}}{e^{2} m^{*}}
\end{array}
$$

As seen above, compensation in TCO should be minimized since the reduced compensation not only increases its donor concentration but also increases its mobility.

\section{Grain boundary scattering}

Most of the available models for grain boundary-limited mobility refer to the works by Petritz, ${ }^{18}$ Tarng, ${ }^{19}$ and Seto. ${ }^{3}$ These theories are based on the model of a barrier induced by charges trapped by states at grain boundaries where there are many defects due to the incomplete chemical bonds. There are typically three different formulas for grain boundary scattering based on this theory in the literature. The one based on thermionic emission from traps on the grain boundaries was developed by Seto $^{3}$ for non-degenerate materials which has the form

$$
\begin{aligned}
\mu_{g}= & \operatorname{Lq}\left(2 \pi m^{*} k_{B} T\right)^{-1 / 2} \exp \left(-\Phi_{\mathrm{b}} / k_{B} T\right) \text { or } \ln \left(\mu_{g} T^{1 / 2}\right) \\
& \sim-T^{-1} .
\end{aligned}
$$

This model is widely cited but is not suitable for degenerate materials. Note that $\mathrm{L}$ is the grain size and $k_{B}$ is Boltzmann constant. As pointed out by Ellmer and Mientus, ${ }^{5}$ the Seto model extended by Werner, ${ }^{20}$ as shown below, can be used to describe the temperature-dependent mobility at lower carrier concentration

$$
\begin{aligned}
& \mu=\frac{\mu_{0} \cdot \mu_{\text {eff }}}{\mu_{0}+\mu_{\text {eff }}} \\
& =\frac{\mu_{0} \cdot \mu_{\text {grain }}}{\mu_{0}+\mu_{\text {grain }} \exp \left(-\frac{\varphi_{b}-\frac{\Delta \varphi_{b}^{2}}{2 K T}}{K T}\right)} \exp \left(-\frac{\varphi_{b}-\frac{\Delta \varphi_{b}^{2}}{2 K T}}{K T}\right),
\end{aligned}
$$

where $\mu_{0}$ is the temperature independent, $\mu_{\text {eff }}$ is the temperature dependent, and $\mu_{\text {grain }}$ is the mobility inside the grain. That developed by Tarng $^{19}$ based on the assumption of Schottky thermionic emission and then followed by Bruneaux et al. $^{4}$ for degenerate materials is

$$
\mu_{\mathrm{g}}=\mathrm{BT} \exp \left(-\Phi_{\mathrm{a}} / \mathrm{k}_{\mathrm{B}} \mathrm{T}\right)
$$

or $\ln \left(\mu_{g} / T\right) \sim-T^{-1}$. Note that $B$ is a constant related to the grain size and electron concentration and $\Phi_{a}$ is the activation energy given by $\Phi_{b}-\left(E_{F}-E_{C}\right)$. If we assume Schottky barrier, the barrier should be high for which a value of $0.5 \mathrm{eV}$ was given by Tarng ${ }^{19}$ for polycrystalline-Si. That probably first used by Zhang and $\mathrm{Ma}^{21}$ for grain boundary scattering is
$\mu_{g}=B T^{-1} \exp \left(-\Phi_{a} / k_{B} T\right)$ or $\ln \left(\mu_{g} T\right) \sim-T^{-1}$, which has been widely used in the literature. ${ }^{9,10}$ With our careful examination, Ref. 21 directly refers to the work by Bruneaux et al., ${ }^{4}$ in which $\mu_{g}=B T \exp \left(-\Phi_{\mathrm{a}} / k_{B} T\right)$ or $\ln \left(\mu_{g} / T\right) \sim-T^{-1}$. However, the temperature dependence of mobility appears in a different form $\left(\ln \left(\mu_{g} T\right) \sim-T^{-1}\right)$ from the original work by Bruneaux et $a l^{4}$ Therefore, the expression used by Zhang and $\mathrm{Ma}^{21}$ is possibly problematic since it is not physically meaningful and they did not mention its inconsistency with the original reference.

When considering grain boundary scattering, we must consider two other physical phenomena. Although they are different in formulism, both are temperature independent. The first one is electron reflection by grain boundaries, which gives the strength of the potential and thickness of the grain boundary. The model for grain boundary scattering based on reflection can be found elsewhere. ${ }^{5,22,23}$ The second phenomenon is quantum mechanical tunnelling. If we consider a simple rectangular barrier of height $U$ and width $W$, the tunnelling probability ${ }^{24}$ can be expressed as

$$
T_{\text {tunn }} \approx \frac{16 E(U-E)}{U^{2}} \exp \left(-2 \sqrt{\frac{2 m^{*}(U-E)}{\hbar^{2}} W}\right) .
$$

Following Holm ${ }^{25}$ for a rectangular barrier of height $E_{B}$ and width $l_{2}$, when a very small applied voltage $\mathrm{V}$ is across a barrier the conductivity by the tunnel effect $\sigma_{\text {tun }}$ can be expressed as ${ }^{26}$

$$
\sigma_{\text {tun }}=\left[L q^{2}\left(2 m^{*} E_{B}\right)^{1 / 2} /\left(h^{2} l_{2}\right)\right] \exp \left[-4 \pi l_{2}\left(2 m^{*} E_{B}\right)^{1 / 2} / h\right],
$$

where $L$ and $h$ are the grain size and Planck constant, respectively. This equation indicates that, for a given free-carrier concentration, the mobility $(\mu=\sigma / q n)$ limited by the tunnel effect is temperature independent but is proportional to the grain size. It should be mentioned that, however, while pure tunnelling is temperature independent in and of itself, but the effective tunnelling-governed electron flow across grain boundaries is temperature-dependent, because the energy distribution of electrons is temperature dependent and electrons with different energies experience barriers of different heights. The last consideration dramatically complicates the theoretical study of grain-boundary scattering for the tunnelling limit (low barriers).

\section{EXPERIMENTAL}

A MBE system (SVT Associates) equipped with a commercial RF oxygen plasma source manufactured by Addon, Inc. and effusion cells for $\mathrm{Zn}$ and $\mathrm{Ga}$ was employed to grow the investigated GZO layers. The critical growth parameters affecting the properties of GZO layers are the substrate temperature $\mathrm{T}_{\text {sub }}$, flux of reactive oxygen, and Ga cell temperature, $\mathrm{T}_{\mathrm{Ga}}$, which in turn controls the $\mathrm{Ga}$ flux during the growth. During growth, the plasma power, $\mathrm{T}_{\text {sub }}$, and $\mathrm{Zn}$ cell temperature $\left(\mathrm{T}_{\mathrm{Zn}}\right)$, were set to be $400 \mathrm{~W}, 400^{\circ} \mathrm{C}$, and $350^{\circ} \mathrm{C}$, respectively, for all the layers used in this investigation. The flux of reactive oxygen, $\mathrm{P}_{\mathrm{O} 2}$, was controlled by $\mathrm{O}_{2}$ supply 
through a mass-flow controller with the corresponding pressure in the growth chamber during growth. In this study, we used three different oxygen pressures $P_{O 2}=4.5 \times 10^{-6}$, $8.0 \times 10^{-6}$, and $1.5 \times 10^{-5}$ Torr, corresponding to metal $(\mathrm{Zn}+\mathrm{Ga})$ rich (reactive oxygen to incorporated $\mathrm{Zn}$ ratio $<1$ ), intermediate or near stoichiometric (reactive oxygen to incorporated $\mathrm{Zn}$ ratio $\approx 1$ ), and oxygen-rich conditions (reactive oxygen to incorporated $\mathrm{Zn}$ ratio $>1: 1$ ), respectively. The reactive oxygen-to-incorporated $\mathrm{Zn}$ ratios were assessed from the growth rate vs. oxygen pressure. ${ }^{27}$ The effects of substrate temperature, $\mathrm{T}_{\text {sub }}$, on the properties of GZO layers have been reported elsewhere. ${ }^{28}$ The best conditions to achieve highly conductive GZO layers are $\mathrm{T}_{\mathrm{sub}}=400^{\circ} \mathrm{C}, \mathrm{P}_{\mathrm{O} 2}=4.5 \times 10^{-6}$ Torr, and $\mathrm{T}_{\mathrm{Ga}}$ $=600{ }^{\circ} \mathrm{C}^{27}$ In order to reveal the effect of growth conditions on the layer microstructure and electron scattering mechanisms, we performed detailed studies of electron transport in two sets of GZO samples using temperature-dependent Hall (TDH) measurements in the van der Pauw configuration. In the first series, we compared electrical properties of the samples grown under metal-rich and oxygen-rich conditions with $\mathrm{T}_{\mathrm{Ga}}=600^{\circ} \mathrm{C}$. In the second series, we varied Ga flux by changing $\mathrm{T}_{\mathrm{Ga}}$ from 425 to $600^{\circ} \mathrm{C}$, while oxygen pressure was kept at $4.5 \times 10^{-6}$ Torr (metal-rich conditions). Rapid thermal annealing (RTA) at a temperature of $\sim 600^{\circ} \mathrm{C}$ in nitrogen environment for 3 min was used for some GZO layers to improve their properties. The structural properties of our GZO layers were characterized by xray diffraction (XRD). For the representatives of GZO layers, scanning transmission electron microscopy (STEM), transmission electron microscopy (TEM), electron energy loss spectroscopy (EELS), and energy dispersive x-ray spectroscopy (EDX) measurements were done on cross-section specimens using an aberration-corrected STEM microscope Titan S-Twin operated at $200 \mathrm{kV}$. The TEM/STEM investigations were focused on characterization of structural defects that could be responsible for carrier scattering and limitation of electron mobility in GZO.

\section{RESULTS AND DISCUSSION}

First, we have studied the general relationship between the microstructure of GZO layers grown under various reactive-oxygen-to-metal ratios and their transport properties at RT. Then, the electron concentration and mobility were investigated for two sets of the layers described in the "Experimental" Section in order to gain insight into electron scattering mechanisms governing the transport in GZO.

\section{Correlation between crystal structure and room-temperature electron mobility in GZO}

STEM and TEM studies revealed that the microstructure of GZO layers strongly depend on $P_{O 2}$, i.e., oxygen-to-metal ratio. Inclined grain boundaries $\left(12^{\circ}\right.$ from the polar $c$-direction indicated by the two arrows) are the dominating extended structural defects in the GZO layers grown under oxygen-rich growth conditions [Figure 1(a)]. We conjecture that they should have strong polar field in $\mathrm{ZnO}$ because the polarization vector pointing into the $c$-direction will have a substantial projection on them and as a consequence, coulombic interaction between free carriers and grain boundaries will reduce electron mobility. The grain size in these

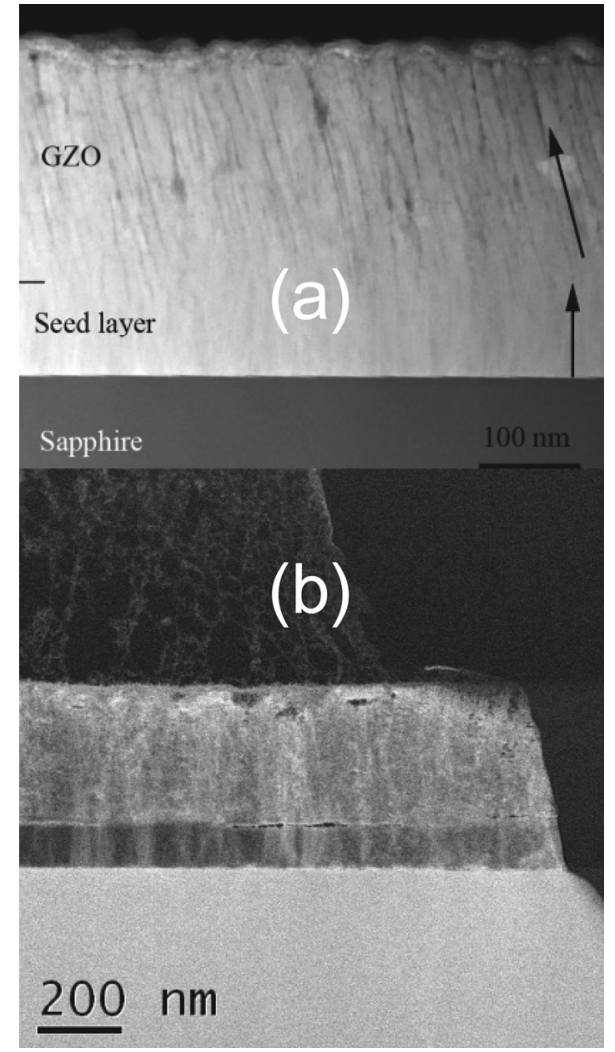

FIG. 1. (a) A representative of STEM images of GZO layers grown under oxygen-rich conditions and (b) LAADF TEM image of a representative GZO layer grown under metal-rich conditions (GZO-1 as shown later). Note: arrows indicating inclined grain boundaries ( $12^{\circ}$ from the polar $c$-direction).

GZO films varies in the range from 10 to $30 \mathrm{~nm}$. Unlike the GZO layers grown under oxygen-rich conditions, the GZO films grown on a-sapphire under metal-rich growth condition have high crystal quality but albeit with some porosity and porous GZO grows on top of the non-porous $\mathrm{ZnO}$ seed layer. Investigation of the nature of pores revealed that these defects form due to nucleation of multiple open-core dislocations in GZO layer. Open-core dislocations have a minor effect on the width of the XRD rocking curves. However, the high concentration of pores masks the position of low-angle grain boundaries in STEM images and consequently makes it difficult to use STEM technique to visualize the grain boundaries by varying electron channelling effect for grains oriented at different angles with respect to the probe direction.

In order to gain accurate information regarding the grain boundaries and grain sizes for GZO layers grown under metal-rich conditions, low angle annual dark field (LAADF) technique was employed. Also the special care was taken about specimen preparation in order to minimize the effect of ion milling on the sample microstructure. As shown in Fig. 1(b), the low-angle grain boundaries in the GZO layer grown under metal-rich conditions mainly propagate from the $\mathrm{ZnO}$ seed layer normally to the substrate plane (parallel to the polar $c$-direction), which is totally different from that for GZO layers grown under oxygen-rich conditions as we discussed above [Figure 1(a)]. Therefore, the grain boundaries in GZO grown under metal-rich conditions are parallel to the $c$-direction that results in much smaller polarization 
field at the interfaces. The average grain sizes in these layers were determined to vary from $30 \mathrm{~nm}$ to $50 \mathrm{~nm}$, which are larger than those for GZO layers grown under oxygen-rich conditions. Owing to the larger grain size and the smaller charge accumulation on low-angle grain boundaries, the mobility limited by grain boundaries in GZO layers grown under metal-rich conditions should be higher than that in GZO layers grown under oxygen-rich conditions.

Figure 2 shows the Hall electron mobility versus either electron concentration [Fig. 2(a)] or grain size [Fig. 2(b)] for the as-grown and annealed GZO layers, for which carrier concentrations varied from $3 \times 10^{20}$ to $12 \times 10^{20} \mathrm{~cm}^{-3}$. Note that the grain size was extracted from the (0002) XRD rocking curves using the Scherrer equation ${ }^{29}$ except the two points with error bars (one for the GZO layer grown under metal-rich conditions and the other one for the GZO layer grown under oxygen-rich conditions), for which grain sizes were determined from STEM/TEM cross-section measurements. The error bar in the mobility is due to the spatial nonuniformity of electrical properties over the wafer. As seen from Fig. 2(a), there is no clear correlation between the electron mobility and the concentration. However, the mobility vs. grain size plot reveals a nearly linear relationship
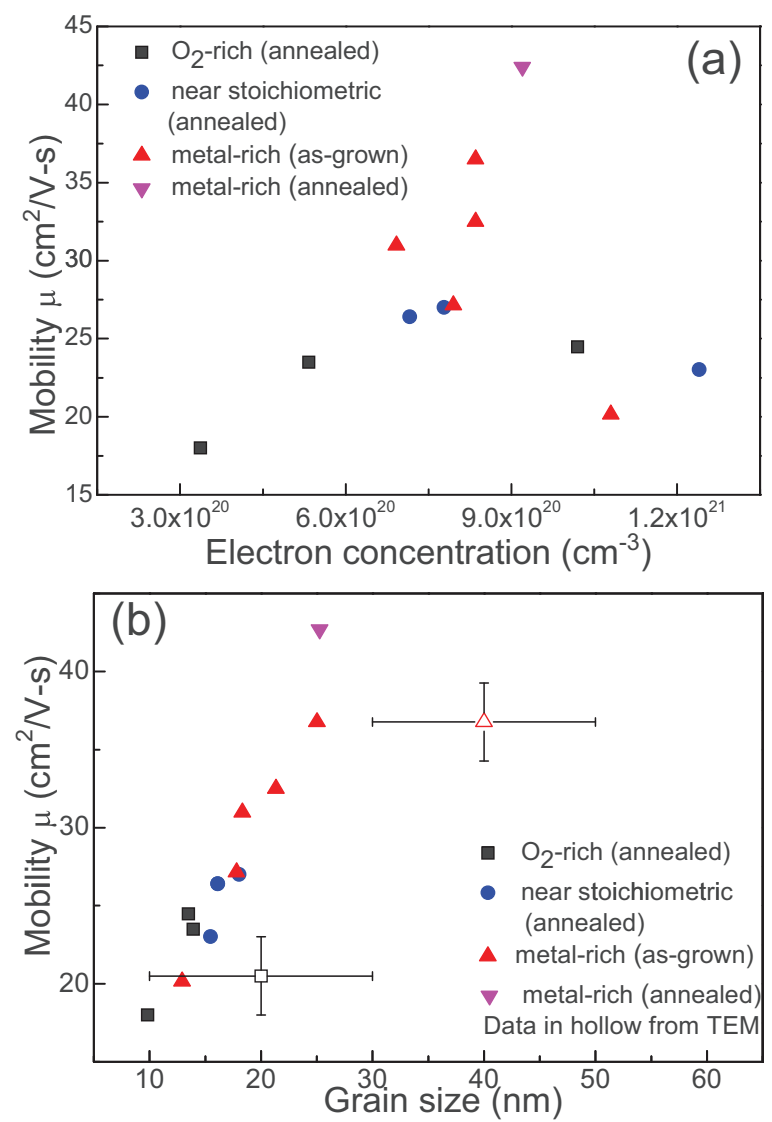

FIG. 2. Hall mobilities vs. (a) electron concentrations and (b) grain sizes for annealed GZO layers with different thicknesses grown under oxygen-rich conditions (squares), annealed GZO layers with different thicknesses grown under near stoichiometric conditions (circles), and as-grown GZO layers with different thicknesses grown under metal-rich conditions (triangles). [Symbols in hollow from STEM/TEM for comparisons; an annealed GZO grown under metal-rich condition (highest $\mu$ ) was also added to see RTA treatment effect.] between these two parameters. At a first glance, the data appear to indicate as if mainly the grain boundary scattering limits the electron transport. However, one should keep in mind that the full picture is quite complicated, which was caused by the differences in oxygen pressures during growth and film thicknesses, etc. The detailed discussion regarding the effects of oxygen pressures, film thickness, and annealing on GZO electrical properties as well as grain sizes can be found elsewhere. ${ }^{27}$ In short, annealing in nitrogen environment significantly improved the electrical properties of GZO grown under oxygen-rich conditions while only minor increase in mobility and electron concentration observed for GZO grown under metal-rich conditions. The difference in the annealing behavior is tentatively attributed to the lower concentration of compensating defects in GZO grown under metal-rich conditions. ${ }^{12}$ For highly conductive GZO with electrical properties comparable to our best GZO grown under metal-rich conditions, Look et al. ${ }^{30}$ reported the existence of Zn-vacancy-related acceptors causing selfcompensation based on positron annihilation measurements and secondary-ion mass spectroscopy (SIMS). This seems consistent with our observation of slight increase in both the electron concentration and mobility upon annealing for one GZO layer grown under metal-rich conditions ( $\mathrm{n}=$ $8.33 \times 10^{20} \mathrm{~cm}^{-3}$ and $\mu=36.7 \mathrm{~cm}^{2} / \mathrm{V} \cdot \mathrm{s}$ in the as-grown sample vs. $\mathrm{n}=9.23 \times 10^{20} \mathrm{~cm}^{-3}, \mu=42.4 \mathrm{~cm}^{2} / \mathrm{V} \cdot \mathrm{s}$ in the annealed one). Note that the annealed sample is actually GZO-1 which will be discussed in more details later. Since the grain size remains virtually unchanged $(\sim 25 \mathrm{~nm})$ before and after annealing as shown in Fig. 2, the increase in both the electron concentration and mobility is unlikely due to the reduced effect of grain boundary scattering but possibly due to the decrease in concentration of Zn-vacancy defects, which is consistent with the increased carrier concentration upon annealing. It must be reiterated that the concentration of compensating defects in our GZO layers grown under oxygen-rich conditions should be much higher than that in GZO layers grown under metal-rich conditions, resulting in a serious compensation in GZO grown under oxygen-rich conditions. ${ }^{12}$ As discussed earlier, grain boundary scattering governed by quantum-mechanical tunneling is virtually temperature-independent, which is the same as that of ionized impurity scattering in the degenerate material. Therefore, the large variation in electron concentrations from $3 \times 10^{20} \mathrm{~cm}^{-3}$ to $9 \times 10^{20} \mathrm{~cm}^{-3}$ and/or in growth conditions could mask the real phenomenon and lead to a wrong conclusion.

\section{Electron transport in GZO grown under oxygen-rich and metal-rich conditions}

The electron transport was studied in greater details using TDH measurements in the van der Pauw configuration for 4 selected GZO layers. Table I summarizes the growth details and sample characteristics.

The TDH data displayed in Fig. 3 clearly show that the carrier concentration is temperature independent for all four samples, indicating their degeneracy. It is worth nothing that the layers with higher carrier concentrations also have higher 
TABLE I. Basic information for 4 selected GZO layers used for TDH measurements.

\begin{tabular}{lccccccc}
\hline \hline No & Thickness (nm) & $\mathrm{P}_{\mathrm{O} 2}\left(10^{-6}\right.$ Torr $)$ & ZnO buffer thickness $(\mathrm{nm})$ & Annealed? & FWHM of $(0002) \mathrm{ZnO}(\mathrm{deg})$ & $\mathrm{RT} \mu\left(\mathrm{cm}^{2} / \mathrm{V} \cdot \mathrm{s}\right)$ & $N\left(10^{20} \mathrm{~cm}^{-3}\right)$ \\
\hline GZO-1 & 290 & 4.5 & $\sim 100$ & Yes & 0.34 & 42.4 \\
GZO-2 & 523 & 4.5 & $\sim 10$ & No & 0.39 & 3.2 \\
GZO-3 & 745 & 15 & $\sim 10$ & Yes & 0.88 & 18.2 \\
GZO-4 & 745 & 15 & $\sim 10$ & No & 1.15 & 7.5 & 3.37 \\
\hline \hline
\end{tabular}

mobilities in the entire temperature range investigated (the order is GZO-1, GZO-2, GZO-3, and GZO-4). GZO-4 has the lowest carrier concentration of around $2 \times 10^{19} \mathrm{~cm}^{-3}$ and the lowest mobility of $5-7 \mathrm{~cm}^{2} / \mathrm{V} \cdot \mathrm{s}$ for temperatures ranging from 15 to $330 \mathrm{~K}$. Upon annealing in nitrogen, this layer
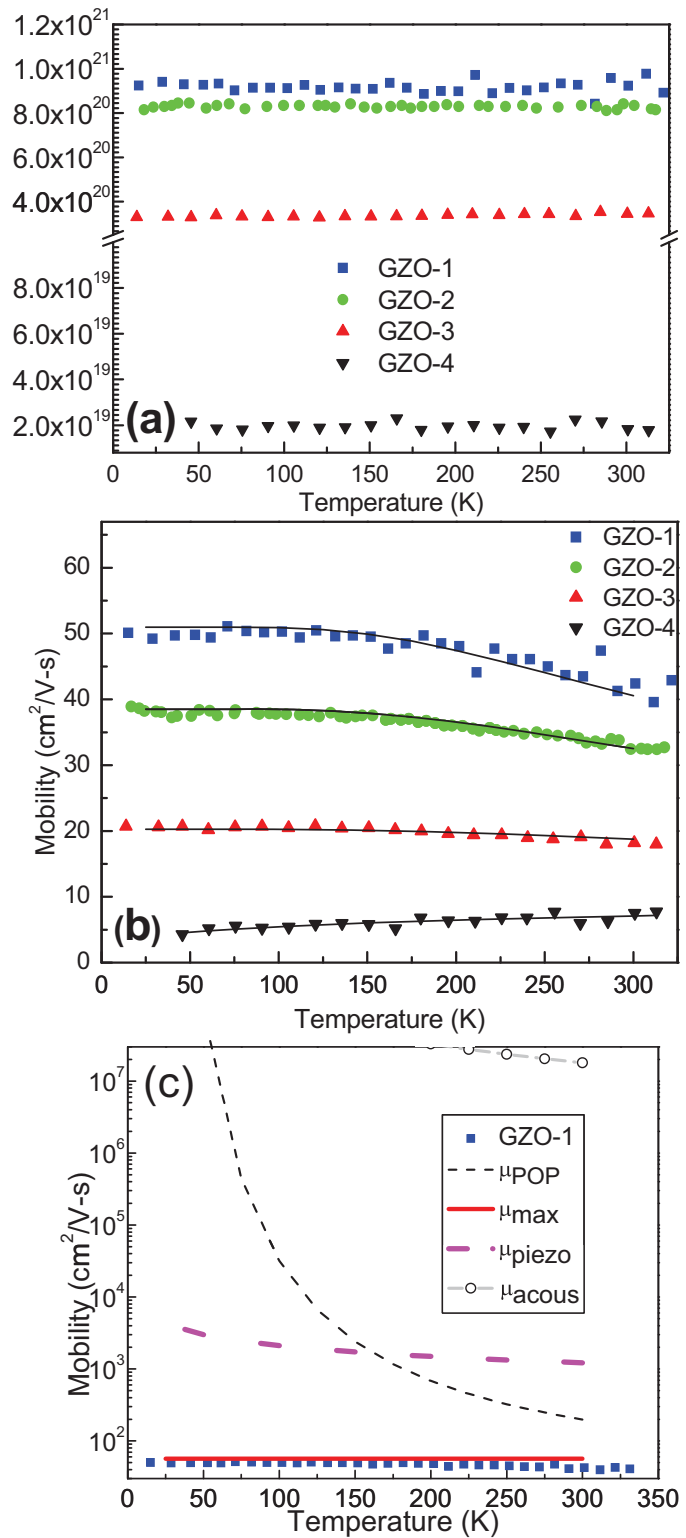

FIG. 3. Temperature dependence of (a) carrier concentration and (b) mobility for GZO-1, GZO-2, GZO-3, and GZO-4 (from top to bottom in both) [Note: solid lines in (b) are fittings. For GZO 1-3, the fittings used a constant for mobility limited by temperature-independent scatterings, and polar optical phonon scattering for temperature-dependent scattering while for GZO-4, it was fitted with power dependence], (c) effects of different scattering mechanisms in GZO-1 indicating ionized impurity scattering and POP scattering is the first and the second dominant mechanisms limiting the RT mobility. (denoted as GZO-3 in Table I) exhibits much higher carrier concentration and mobility of $\sim 3.4 \times 10^{20} \mathrm{~cm}^{-3}$ and 18-to$21 \mathrm{~cm}^{2} / \mathrm{V} \cdot \mathrm{s}$, respectively, but these figures are still well below those for the GZO layers grown under metal-rich conditions (GZO-1 and 2). GZO-1 has a slightly higher carrier concentration and mobility possibly because of the thicker undoped $\mathrm{ZnO}$ buffer layer grown at $600^{\circ} \mathrm{C}$ and/or possibly the lower concentration of defect-related compensating acceptors as compared to GZO-2. Note that the thicker $\mathrm{ZnO}$ buffer layer grown at $600^{\circ} \mathrm{C}$ enhances the structural perfection giving rise to a slightly narrower FWHM of XRD (0002) peak. From the temperature dependent resistivities (not shown here), GZO-1 and GZO-2 showed the metallic behavior. The lowest resistivity achieved at low temperatures is $\sim 1.3 \times 10^{-4} \Omega \cdot \mathrm{cm}$. GZO-3 exhibited a similar temperature dependence as GZO-1 and GZO-2 but its resistivity is much higher $\left(\sim 9.2 \times 10^{-4} \Omega \cdot \mathrm{cm}\right)$, while GZO-4 showed a semiconductor-like behavior (negative temperature coefficient of resistivity).

We have fitted the measured temperature dependent mobilities for samples GZO 1-4 as shown in Fig. 3(b). The un-annealed GZO layer grown under oxygen-rich conditions (GZO-4 sample with the lowest carrier concentration of $2 \times 10^{19} \mathrm{~cm}^{-3}$ among the samples considered in this Section) exhibits a $\mu \sim T^{0.24}$ temperature dependence [Fig. 3(b)]. Temperature-activated mobility of GZO-4 can be considered as a characteristic of grain boundary scattering based on thermionic effect, ${ }^{3,5}$ representing a higher barrier at grain boundaries in the material with lower electron concentration.

Samples GZO-1, GZO-2, and GZO-3 show the temperature dependences of mobility which drastically different from that for the GZO 4 layer. Their mobilities initially increase when temperature decreases down to $\sim 150 \mathrm{~K}$. At lower temperatures (LTs), mobility in these samples becomes virtually invariant. The temperature dependent mobilities measured in GZO-1, GZO-2, and GZO-3 were theoretically fitted based on Matthiessen's rule as $1 / \mu=1 / \mu_{\mathrm{Ti}}+1 / \mu_{\mathrm{pop}}$, where $\mu_{T i}$ is a constant representing temperature-independent scattering events, which include ionized impurity scattering $\mu_{i i}$ and grain boundary scattering $\mu_{\mathrm{gb}}$ governed by the tunnel effect, and $\mu_{p o p}$ is the temperature dependent POP scattering (see Fig. 3(b)). The results of fitting are summarized in Table II.

Using the values equal to $\sim 0.39 m_{0}, 0.37 m_{0}$, and $0.33 m_{0}$ measured by Ruske $e t$ al. ${ }^{8}$ for electron effective mass in $\mathrm{ZnO}-$ based TCO with carrier concentrations similar to those in the GZO 1, GZO 2, and GZO 3 layers, based on Eq. (1) we calculated RT $\mu_{p o p}$ to be $\sim 198,210$, and $250 \mathrm{~cm}^{2} / \mathrm{V} \cdot \mathrm{s}$ in GZOs 1 to 3, respectively. (Note: $e^{*} / e=0.62, M=2.135 \times 10^{-23} \mathrm{~g}$, $V_{a}=2.38 \times 10^{-23} \mathrm{~cm}^{3}$ in Eq. (1) for POP scattering). The calculated values for POP scattering-limited mobility at RT 
TABLE II. Results of fitting to the temperature-dependent mobility for GZO 1-3 based on Matthiessen's rule $1 / \mu=1 / \mu_{\mathrm{Ti}}+1 / \mu_{\text {pop }}$.

\begin{tabular}{lccc}
\hline \hline No & $\mu\left(\mathrm{cm}^{2} / \mathrm{V} \cdot \mathrm{s}\right)$ & $\mu_{\mathrm{Ti}}\left(\mathrm{cm}^{2} / \mathrm{V} \cdot \mathrm{s}\right)$ & $\mathrm{RT} \mu_{\text {pop }}\left(\mathrm{cm}^{2} / \mathrm{V} \cdot \mathrm{s}\right)$ \\
\hline GZO-1 & $\sim 40.6$ & 51 & 198 \\
GZO-2 & $\sim 32.5$ & 38.5 & 210 \\
GZO-3 & $\sim 18.7$ & 20.3 & 250 \\
\hline \hline
\end{tabular}

are comparable to the reported mobility limited by lattice scattering ( $\sim 200 \mathrm{~cm}^{2} / \mathrm{V} \cdot \mathrm{s}$, Ref. 5) and consequently demonstrate that other phonon scatterings which are temperaturedependent are negligible compared with POP scattering in $\mathrm{ZnO}$ due to its highly polar nature as discussed earlier. Since other mechanisms used in the simulations are to a first extent temperature-independent in these very highly doped samples, POP scattering is suggested to be the mechanism responsible for the temperature-dependence, which further reduces the mobility for GZO 1-3 at T > 150 K.

Based on the Brooks-Herring formula for degenerate materials, the $\mu_{\max }$ (Eq. (2)) representing the maximum mobility limited by ionized impurity scattering with no compensation (mobility at $0 \mathrm{~K}$ ) were calculated to be $56.7,61.5$, and $87.5 \mathrm{~cm}^{2} / \mathrm{V}$.s for GZO 1-to-3 layers, respectively. Note that the dielectric constant $\varepsilon_{0}$ and ionization charge $Z$ are 8.12 and 1. ${ }^{11}$ However, the simulated mobilities limited by temperatureindependent scattering events are 51,39 , and $20 \mathrm{~cm}^{2} / \mathrm{V} \cdot \mathrm{s}$ for GZO 1-to-3, respectively. Based on Matthiessen's rule, the mobilities limited by both compensation if present and grain boundary scattering based on tunnelling can be calculated as $507.3,106.6$, and $27.6 \mathrm{~cm}^{2} / \mathrm{V} \cdot \mathrm{s}$ for GZO 1-to-3, respectively. We should mention here that it is extremely difficult to separate out the contribution from grain-boundary scattering governed by tunnelling and compensation.

The mobility of $507.3 \mathrm{~cm}^{2} / \mathrm{V} \cdot \mathrm{s}$ limited by grain boundary scattering and compensation if compensation present in GZO1 is $\sim 9$ times higher than the mobility limited by ionized impurity scattering in the whole investigated temperature range and consequently the grain boundary scattering and compensation have a relatively minor effect and thus both can be neglected. In this case, ionized impurity scattering and POP scattering is the first and the second dominant mechanisms limiting the RT mobility, respectively. Figure 3(c) graphically summarizes the theoretical effects of different scattering mechanisms for GZO-1. For GZO-2, the mobility set by grain boundary scattering based on tunnelling and compensation if present $\left(106.6 \mathrm{~cm}^{2} / \mathrm{V} \cdot \mathrm{s}\right)$ is larger by a factor of $\sim 1.73$ times higher than the mobility limited by ionized impurity scattering $\left(61.5 \mathrm{~cm}^{2} / \mathrm{V} \cdot \mathrm{s}\right)$ in the whole temperature range investigated. The stronger effect of grain boundary scattering and compensation in GZO-2 is consistent with its slightly larger rockingcurve width $\left(0.39^{\circ}\right.$ vs. $\left.0.34^{\circ}\right)$ and slightly lower electron concentration of $\sim 8.4 \times 10^{20} \mathrm{~cm}^{-3}$, indicating a somewhat smaller grain size and, plausibly, a higher compensation ratio as well. By comparing GZO-2 with GZO-1, the probability of existence of compensation caused by defects such as Znvacancy acceptors is greater. If we assume no compensation in GZO-2, the grain boundary scattering would have a larger effect than POP scattering at RT (106.6 vs. $\left.210 \mathrm{~cm}^{2} / \mathrm{V} \cdot \mathrm{s}\right)$. No matter which one is stronger, ionized impurity scattering is still the first dominant mechanisms limiting the mobility in the entire temperature range $15-330 \mathrm{~K}$. However, the grain boundary scattering may compete with POP scattering in the practically important temperature range around RT depending on the GZO film quality (the higher the structural quality, the smaller the contribution of grain boundaries).

For GZO-3, the mobility limited by the grain boundary scattering and compensation $\left(27.6 \mathrm{~cm}^{2} / \mathrm{V} \cdot \mathrm{s}\right)$ is much lower than that limited by ionized impurity scattering $\left(87.5 \mathrm{~cm}^{2} / \mathrm{V} \cdot \mathrm{s}\right)$, which is consistent with the predicted higher compensation by Demchenko et al. ${ }^{12}$ and smaller grain size revealed by STEM and XRD characterization in the GZO layers grown under oxygen-rich conditions. However, it is difficult to delineate and quantify the contributions from grain boundary scattering and compensation in GZO-3. But it is obvious that, when the grain size becomes smaller and electron concentration reduces, the effect of grain boundary scattering becomes stronger, while the contribution of POP scattering weakens. To make the situation worse, the donor compensation provides a "positive feedback" to the grain boundary scattering. For a given $\mathrm{Ga}$ concentration and a given average grain size, the higher the compensation is the lower the Fermi energy is, i.e., the higher the barriers are and consequently the stronger the grain boundary scattering is. Note that the smaller grain sizes for GZO grown under oxygenrich conditions are definitely caused by excess oxygen during growth compared with that for GZO grown under metal-rich conditions. In other words, the smaller the grain sizes are, the larger the volume ratio of grain boundaries is and consequently more defects could be trapped in the boundary regions. As the measured electron concentration is much lower in GZO grown under oxygen-rich conditions, compensation caused by the increased defects is more likely. Wong et al. ${ }^{31}$ reported that excess oxygen will lead to compensation while more excess oxygen will lead to structural changes which increase the grain boundary scattering. This reasoning is consistent with our observations and analysis. In short, metal-rich conditions instead of oxygen-rich conditions are the must to achieve high mobility and high electron concentration in GZO. However, as suggested by data presented in Fig. 2, in order to push the mobility to the value limited by scattering only on ionized impurities and polar optical phonons, large grain size is required even for the material grown under metal-rich conditions.

\section{Effect of Ga flux via changing Ga cell temperature ( $\left.T_{G a}\right)$}

Table III lists the growth conditions and major structural and electric properties of GZO layers discussed in this Section. These samples were grown under metal-rich conditions $\left(\mathrm{P}_{\mathrm{O} 2}=4.5 \times 10^{-6}\right.$ Torr $)$ with varying $\mathrm{Ga}$ cell temperatures. As seen from the table, when $\mathrm{T}_{\mathrm{Ga}}$ was increased from 425 to $600^{\circ} \mathrm{C}$, the carrier concentration increases from $\sim 6 \times 10^{18}$ $\mathrm{cm}^{-3}$ to $9 \times 10^{20} \mathrm{~cm}^{-3}$. RT mobility first increases from $\sim 34$ to $40 \mathrm{~cm}^{2} / \mathrm{V} \cdot \mathrm{s}$, when $\mathrm{T}_{\mathrm{Ga}}$ was increased from 425 to $450{ }^{\circ} \mathrm{C}$ and then the RT mobility remains virtually unchanged, when $\mathrm{T}_{\mathrm{Ga}}$ was further increased to $600^{\circ} \mathrm{C}$.

The results of TDH measurements for 3 selected GZO layers listed in Table III are shown in Fig. 4. The evolution 
TABLE III. Basic information for 3 selected GZO layers used for TDH measurements.

\begin{tabular}{lccccccc}
\hline \hline No & Thickness $(\mathrm{nm})$ & $\mathrm{T}_{\mathrm{Ga}}\left({ }^{\circ} \mathrm{C}\right)$ & $\mathrm{ZnO}$ buffer thickness $(\mathrm{nm})$ & Annealed? & FWHM of (0002) ZnO $(\mathrm{deg})$ & $\mathrm{RT} \mu\left(\mathrm{cm}^{2} / \mathrm{V} \cdot \mathrm{s}\right)$ & $\mathrm{n}\left(10^{20} \mathrm{~cm}^{-3}\right)$ \\
\hline GZO-1 & 290 & 600 & $\sim 100$ & Yes & 0.34 & 42.4 \\
GZO-5 & 350 & 450 & $\sim 10$ & No & 0.325 & 9.2 \\
GZO-6 & 290 & 425 & $\sim 10$ & No & 0.319 & 0.1 & 34.3 \\
\hline \hline
\end{tabular}

of temperature dependences of mobility with electron concentration here is similar to what we discussed above, namely in Section "Electron transport in GZO grown under oxygen-rich and metal-rich conditions." Figure 4 clearly shows that, as the electron concentration reduces, the signature of the grain-boundary scattering governed by the thermionic effect (mobility increases with temperature) emerges in the temperature dependence of mobility. As the grain boundary scattering governed by the quantum tunnelling (temperature-independent) contributes more for GZO with higher electron concentration, the temperature dependence for GZO-5 is much weaker than that for GZO-6. GZO-6 exhibits a $\mu \sim T^{0.62}$ temperature dependence. While in GZO-1, only grain boundary scattering governed by quantum tunnelling contributes and therefore it is temperature-independent. Note that we have previously demonstrated that grain boundary scattering is negligible in GZO-1. It must be pointed out that dislocation scattering has a similar temperature-dependence as that for grain boundary scattering governed by the thermi-
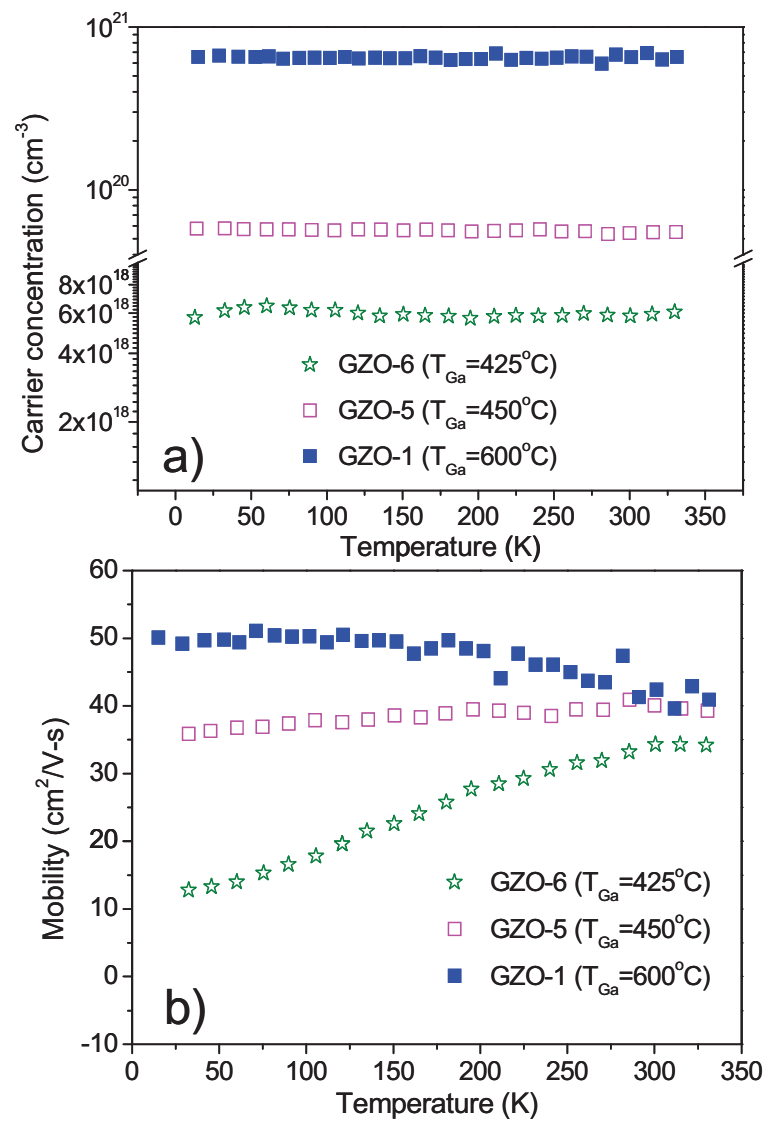

FIG. 4. Temperature dependence of (a) carrier concentration and (b) mobility for GZO-1, GZO-5, and GZO-6. onic effect but the mobility limited by dislocation scattering is at least 30 times higher than the experimental data based on the equation and constants in Ref. 15. Dislocation scattering is thereby not considered here. Since GZO-1, GZO-5, and GZO-6 have comparable FWHMs of (002) XRD rocking curves and thus comparable grain sizes, the change in the behavior of temperature-dependent mobility can be mainly attributed to different barrier heights in the layers with different electron concentrations. The barrier heights for GZO-5 and GZO-6 were estimated using Eq. (4) based on the Seto ${ }^{3}$ model extended by Werner ${ }^{20}$ to be $0.3 \mathrm{meV}$ and $8 \mathrm{meV}$, respectively. Although the values of barrier heights, especially the one for GZO-5, may not be very accurate, it offers a viable explanation for the observed evolution of temperature dependences of mobility with electron concentration.

One interesting observation is that the GZO-5 layer having a slightly higher carrier concentration than that in GZO-4 shows similar temperature dependence of mobility to that of GZO-4 [compare Figs. 3(b)] but with a much higher mobility value within the entire temperature range studied. The narrower rocking curve, by a factor of $\sim 3.5$, and the expected lower compensation ratio in GZO-5 grown under metal-rich conditions are consistent with this observation and the above discussion, indicating that the mobility limited by grain boundary scattering and compensation in GZO-5 grown under metal-rich conditions should be higher than that in GZO-4 grown under oxygen-rich conditions.

\section{CONCLUSIONS}

In an effort to determine and account for the temperature dependence of electron mobility in GZO ( $\mathrm{ZnO}: \mathrm{Ga}$ ) layers, we investigated the electron transport in a comprehensive set of samples grown by MBE on a-plane sapphire substrates with varying oxygen-to-metal ratio and Ga flux, and correlated the electron concentration and temperature dependence of mobility with the microstructure of these layers. For heavily doped GZO with carrier concentrations above $10^{20} \mathrm{~cm}^{-3}$, the temperature dependence of mobility measured in the range $15-330 \mathrm{~K}$ is well described by the Matthiessen's rule with mobility being limited by POP scattering, and a temperature-independent mobility limited by ionized impurity scattering, compensation of $\mathrm{Ga}$ donors with acceptor defects, and electron scattering by low-angle grain boundaries limited by quantum-mechanical tunnelling. Our data indicate that ionized impurity scattering is the dominant mechanism limiting the mobility in the range $15-330 \mathrm{~K}$ for GZO layers with high structural quality grown under metalrich conditions, which have porous features as well as lowangle grain boundaries parallel to the $c$-axis and relatively large average grain size of $20-25 \mathrm{~nm}$, determined by XRD 
$(30-50 \mathrm{~nm}$ by TEM). For these GZO layers grown under metal-rich conditions, POP scattering is the mechanism responsible for the temperature-dependence for $\mathrm{T}>150 \mathrm{~K}$ and thereby POP scattering cannot be neglected especially at RT. For the sample with $\mathrm{n}=\sim 9 \times 10^{20} \mathrm{~cm}^{-3}$ and LT mobility of $\sim 51 \mathrm{~cm}^{2} / \mathrm{V} \cdot \mathrm{s}$ at low temperatures, both grain boundary scattering and compensation if present are negligible due to their very minor effects. In contrast, for heavily doped GZO layers grown under oxygen-rich conditions, which have inclined grain boundaries and relatively small grain sizes of $10-20 \mathrm{~nm}$ determined by x-ray diffraction $(10-30 \mathrm{~nm}$ by TEM), the compensation and grain boundary scattering became dominant. The high donor compensation in these layers is caused presumably by $\left(\mathrm{Ga}_{\mathrm{Zn}}-\mathrm{V}_{\mathrm{Zn}}\right)$ complexes having the lowest formation energy in degenerate GZO grown under oxygen-rich conditions. The evolution of temperature dependences of mobility when the electron concentration reduces from $10^{20} \mathrm{~cm}^{-3}$ to $10^{18} \mathrm{~cm}^{-3}$ indicates that not only the contribution of grain-boundary scattering becomes stronger but also that the electron transport across boundaries changes from quantum-mechanical tunnelling to thermionic emission. In short, although the significance order of scattering mechanisms can differ for GZO layers with different growth conditions, metal-rich growth conditions, which can produce films with minimal deleterious effects by compensation and grain boundaries, are imperative for attaining high mobilities and high electron concentrations.

\section{ACKNOWLEDGMENTS}

This work was supported by DOE (Project No. DE-FG02-08ER46547). We thank Dr. D. O. Demchenko for useful discussions.

${ }^{1}$ H. Y. Liu, V. Avrutin, N. Izyumskaya, Ü. Özgür, and H. Morkoç, Superlattices Microstruct. 48, 458 (2010).

${ }^{2}$ H. L. Hartnagel, A. L. Dawar, A. K. Jain, and C. Jagadish, Semiconducting Transparent Thin Films (Institute of Physics Publishing, Philadelphia, 1995).

${ }^{3}$ J. Y. W. Seto, J. Appl. Phys. 46, 5247 (1975).
${ }^{4}$ J. Bruneaux, H. Cachet, M. Froment, and A. Messad, Thin Solid Films 197, 129 (1991).

${ }^{5}$ K. Ellmer and R. Mientus, Thin Solid Films 516, 5829 (2008).

${ }^{6}$ T. Minami, S. Suzuki, and T. Miyata, Mater. Res. Soc. Symp. Proc. 666, F1.3.1 (2001).

${ }^{7}$ J. Steinhauser, S. Faÿ, N. Oliveira, E. Vallat-Sauvain, and C. Ballif, Appl. Phys. Lett. 90, 142107 (2007).

${ }^{8}$ F. Ruske, A. Pflug, V. Sittinger, B. Szyszka, D. Greiner, and B. Rech, Thin Solid Films 518, 1289 (2009).

${ }^{9}$ J. J. Robbins, J. Harvey, J. Leaf, C. Fry, and C. A. Wolden, Thin Solid Films 473, 35 (2005).

${ }^{10}$ B. D. Ahn, S. H. Oh, H. J. Kim, M. H. Jung, and Y. G. Ko, Appl. Phys. Lett. 91, 252109 (2007).

${ }^{11}$ D. C. Look, K. D. Leedy, D. H. Tomich, and B. Bayraktaroglu, Appl. Phys. Lett. 96, 062102 (2010).

${ }^{12}$ D. O. Demchenko, B. Earles, H. Y. Liu, V. Avrutin, N. Izyumskaya, Ü. Ozgür, and H. Morkoç, Phys. Rev. B 84, 075201 (2011).

${ }^{13}$ D. S. Ginley, Handbook of Transparent Conductor (Springer, 2010), Chap. 7.

${ }^{14} \mathrm{~K}$. Ellmer, A. Klein, and B. Rech, Transparent Conductive Zinc Oxide: Basics and Application in Thin film Solar Cells (Springer, 2008), Chap. 3.

${ }^{15}$ Y. S. Jung, O. V. Kononenko, and W.-K. Choi, Solid State Commun. 137, 474 (2006).

${ }^{16}$ K. Ellmer and R. Mientus, Thin Solid Films 516, 4620 (2008).

${ }^{17}$ H. Morkoç, Handbook on Nitride Materials and Devices (Wiley, 2008), Vol. 2, Chap. 3.

${ }^{18}$ R. L. Petritz, Phys. Rev. 104, 1508 (1956).

${ }^{19}$ M. L. Tarng, J. Appl. Phys. 49(7), 4069 (1978).

${ }^{20} \mathrm{~J}$. H. Werner, Polycrystalline Semiconductors III-Physics and Technology (Scitec, Zug, Switzerland, 1993), p. 213.

${ }^{21}$ D. H. Zhang and H. L. Ma, Appl. Phys. A 62, 487 (1996).

${ }^{22}$ S. Riedel, J. Röber, and T. Geßner, Microelectron. Eng. 33, 165 (1997).

${ }^{23}$ S. K. Vasheghani Farahani, T. D. Veal, P. D. C. King, J. Zúniga-Pérez, V. Munoz-Sanjosé, and C. F. McConville, J. Appl. Phys. 109, 073712 (2011).

${ }^{24}$ S. M. Sze and K. K. Ng, Physics of Semiconductor Devices, 3rd ed. (Wiley, 2007), Chap. 1.

${ }^{25}$ R. Holm, J. Appl. Phys. 22, 569 (1951).

${ }^{26}$ M. L. Addonizio, A. Antonaia, G. Cantele, and C. Privato, Thin Solid Films 349, 93 (1999).

${ }^{27}$ H. Y. Liu, V. Avrutin, N. Izyumskaya, M. A. Reshchikov, Ü. Özgür, and H. Morkoç, Phys. Status Solidi RRL 4, 70 (2010).

${ }^{28}$ H. Y. Liu, X. Li, S. Liu, X. Ni, V. Avrutin, N. Izyumskaya, Ü. Özgür, M. A. Reshchikov, and H. Morkoç, Proc. SPIE 7939, 79392E (2011).

${ }^{29}$ Y. K. Fu, C. H. Kuo, C. J. Tun, C. W. Kuo, W. C. Lai, G. C. Chi, C. J. Pan, M. C. Chen, H. F. Hong, and S. M. Lan, J. Cryst. Growth 310, 4456 (2008).

${ }^{30}$ D. C. Look, K. D. Leedy, L. Vines, B. G. Svensson, A. Zubiaga, F. Tuomisto, D. R. Doutt, and L. J. Brillson, Phys. Rev. B 84, 115202 (2011).

${ }^{31}$ L. M. Wong, S. Y. Chiam, J. Q. Huang, S. J. Wang, J. S. Pan, and W. K. Chim, Appl. Phys. Lett. 98, 022106 (2011). 